\section{Parental high concern and adolescent-onset anorexia nervosa}

\author{
A case-control study to investigate direction of causality
}

PHILIP SHOEBRIDGE and SIMON G. GOWERS

\author{
Background Robust evidence that \\ anorexia nervosa is preceded rather than \\ accompanied by high-concern \\ (overprotective) parenting is limited.
}

\begin{abstract}
Aims To look for evidence of parental high concern occurring before any onset of disorder.
\end{abstract}

\begin{abstract}
Method Forty consecutive referrals of adolescent girls with DSM-III-R anorexia nervosa were compared with matched controls using obstetric records and maternal interviews.
\end{abstract}

Results Index mothers reported higher rates of: near-exclusive child care $(P=0.02)$, infant sleep difficulties $(P=0.018)$, severe distress at first regular separation ( $P=0.048)$, high maternal trait anxiety levels $(P=0.008)$ and later age for first sleeping away from home $(P=0.009)$. More index families had experienced a severe obstetric loss prior to their daughter's birth $(P=0.066)$.

Conclusions This study lends evidence to the clinical contention that high-concern parenting in infancy is associated with the later development of anorexia nervosa. This may derive, in part, from aspects of unresolved grief.

Declaration of interest P.S. was supported by a training grant from the WellcomeTrust.
Shaw \& Garfinkel (1990) have discussed the methodological difficulties in researching aetiological factors of anorexia nervosa. Of particular relevance to studies that implicate individual or familial psychological factors is the reliance on subjective, uncorroborated, retrospective information. Prospective research designs have proved impractical. Overprotective/high-concern parental behaviour has been implicated frequently in the aetiology of the disorder but few studies employ methodologies that can address the issues of cause or effect. Hence, we considered the following questions in relation to normal controls:

(a) Do parents of adolescents with anorexia nervosa report more factors indicative of high-concern parenting attitudes and behaviour in their daughter's early infancy and middle childhood?

(b) Have index parents experienced significantly more previous severe obstetric losses, incorporating: perinatal and infant deaths, severe birth defects, multiple miscarriages, second- or third-trimester miscarriages and terminations?

(c) Are maternal trait anxiety levels higher?

\section{METHOD}

The index subjects with anorexia were recruited from 40 consecutive referrals (from within a regional catchment area) to a general-purpose adolescent service specialising in the treatment of eating disorders. All subjects were female, caucasian and satisfied full DSM-III-R criteria for anorexia nervosa (American Psychiatric Association, 1987). Their mean age was 16.9 years, with a mean length of illness of 15 months, 75\% were treated as out-patients.

Matching with normal controls was achieved individually by pairing for age, gender, ethnicity and maternal-child age difference using a large general practitioner (GP) age/gender register. The GP practice was selected to approximately match the occupational grouping profile found in the anorectic group (Market Research Society, 1991). Exclusion criteria included any lifetime child psychiatric diagnosis, and having scores on the Eating Attitudes Test-26 (EAT-26; Garner et al, 1982) of more than 17 (Wood et al, 1992).

\section{Sources of data}

Maternal obstetric record

The obstetric notes of cases and controls were obtained and photocopies of the records were made. All references to the subject's name were masked, enabling these to be scored blind. These records were then rated for details of the mother's previous obstetric history and the subject's pregnancy. Categorical scoring systems were developed for qualitative variables. Previous obstetric histories were recorded using standard definitions of miscarriage, stillbirths and early neonatal, neonatal, perinatal and infant deaths (Forfar, 1984). References to previous complicated deliveries or births of children with severe birth defects were also noted.

\section{Maternal structured clinical interview covering the subject's pregnancy, early infancy and childhood}

This elicited information relating to: past obstetric history, including fertility worries; marital difficulties in the two years before the birth of their daughter and for the following five years; division of caring duties for the baby/infant between their main carers; length of breast-feeding; maternal postnatal depressive illness; infant feeding problems, focusing on severe faddiness and behavioural difficulties at meal times (Marchi \& Cohen, 1990); duration of nursing the newborn in the parent's room; infant sleeping difficulties, particularly coming into the parents' bed against the parents' wishes; consulting child-care professionals in the first few months; worries regarding developmental difficulties; perceived susceptibility to illness; experience of life-threatening illnesses in the first five years; degree of distress experienced by mothers and exhibited by their daughters when they were left on a regular basis (child-minder, nursery or school); age when first allowed to stay overnight away from home. Data were mainly recorded as verbatim responses. Scoring systems were developed to enable blind categorical ratings. 


\section{A maternal self-report anxiety questionnaire}

The State-Trait Anxiety Inventory (STAI; Spielberger, 1983), was used.

\section{Statistical analysis}

Categorical data were analysed using Fisher's exact test or $\chi^{2}$ comparisons between the two groups. Continuously distributed data, such as the STAI scores, were compared using $t$-tests. Statistical procedures were carried out either using the SPSS/PC software package (version 5.0) or INSTAT2, employing two-tailed statistical tests.

\section{RESULTS}

\section{Recruitment and matching}

Forty-six consecutively referred patients diagnosed as having DSM-III-R anorexia nervosa were identified, two were excluded because of unavailability of parents for interview. The refusal rate was $9 \%(4 / 44)$. Fifty-three matched normal control subjects were invited to participate: six were uncontactable; one was excluded (because of diagnosis of a psychiatric condition); and six refused $(13 \%)$.

In addition to the excellent individual matching for the prospectively chosen demographic variables (see Table 1), we also found a number of other potentially important confounding variables that were almost perfectly matched: children's birth order; birth histories; parental marital status at the time of becoming pregnant; frequency and severity of marital difficulties both before and after the birth of the index subjects; and maternal puerperal smoking habits.

Table I Matching of index subjects and their controls

\begin{tabular}{lll}
\hline & Anorectic group & Control subjects \\
\hline Gender & All female & All female \\
Race & All caucasian & All caucasian \\
Subject's age (years) & Mean=I7.I (s.d.=I.88) & Mean=17.5 (s.d.=I.74) \\
& Range I2-20 years & Range 19-4I years \\
Mother-daughter age difference & Mean=27.I (s.d.=4.63) & Mean=27.30 (s.d.=4.66) \\
& Range I8-4I years & Range 19-20 years \\
Occupation grouping categories' & I4 A\&B & I4 A\&B \\
& 26 CI C2 D E & 26 CI C2 D E \\
\hline
\end{tabular}

I. The Market Research Society (1991) child

Index mothers cerebral palsy

3 Stillbirth: unexpected; at term; anencephalic; female

8 Infant death: cot death, female child aged 9 months

9 Late termination: termination of pregnancy at 14 weeks

Control mothers syndrome after 2 days

3 Late miscarriage at 16 weeks

\section{Data capture rates}

We obtained medical archival information on $95 \%$ of the subjects with anorexia nervosa $(38 / 40)$ and $90 \%$ of the normal controls $(36 / 40)$, one of these relying on GP rather than complete hospital records. One set of the patients' notes was reported lost and another proved unobtainable. In these instances the maternal interview obstetric history data were used.

Semi-structured interview data were obtained on all subjects. Of the normal control subjects $97.5 \%$ (39/40) completed an EAT-26 $($ mean $=3.03$, s.d. $=3.11$, range $=0-17)$. One control subject scored 17 and all others scored 10 or below.

Table 2 Listing of individual cases who had experienced severe obstetric losses prior to the birth of their

I Early neonatal death: male 26-week premature baby died after 5 minutes

2 Severe birth defect. Firstly a miscarriage at 13 weeks. Subsequently a female child born with severe

4 Early neonatal death: male 30-week premature baby born alive but died on the day of birth

5 Early neonatal death: female 36-week premature baby born alive but died of respiratory distress syndrome after 4 days. Prior to this the subject had a miscarriage at 21 weeks; this was also female.

6 Late termination: termination of pregnancy at 16 weeks. The father of this subject had effectively lost his three children by an earlier marriage because his ex-wife's family had joined a religious sect

7 Early neonatal death: male child born at term but died of meconium aspiration after 3 days

10 Multiple miscarriage: three sequential miscarriages at 8,12 and 12 weeks

I Early neonatal death: male 26-week premature baby born alive but died of respiratory distress

2 Multiple miscarriage: three sequential miscarriages at 12, 20 and 10 weeks

\section{Data from the obstetric and maternity case notes}

\section{Preconceptual factors}

Five of the index cases and eight control case notes recorded a previous first-trimester miscarriage. Fifteen per cent $(6 / 40)$ of the index mothers had experienced a perinatal or infant death prior to the birth of their child with anorexia nervosa, compared with $2.5 \%(1 / 40)$ of their matched normal controls $(P=0.108)$. In total, 10 of the group with anorexia nervosa had experienced a prior severe obstetric loss $(25 \%)$ compared with three $(7.5 \%)$ of their matched controls $(P=0.0661)$ (see Table 2). In all but one of these cases the subjects with anorexia nervosa were the next-born female child.

The suggestion of unresolved loss is attested to by the verbatim reports of these mothers. Firstly, the quotes of the mothers with anorexia nervosa:

Case I. "It didn't hit me until the new year. I was really devastated. My attitude to children changed dramatically. From not being fussed I went to really wanting children. I got pregnant earlier than the doctors wished."

Case 2. After the birth of her healthy index child, following a second-trimester miscarriage and the birth of a severely handicapped daughter this mother recalled: "When $\mathrm{H}$ was born I didn't like her, she was revoltingly normal. I couldn't bring 
myself to love her, I couldn't love her. My husband had to force me to go and visit her in the SCBU (Special Care Baby Unit)".

Case 3. "I was really numb for a few months. l'd already lost my mother through suicide, nothing could be as bad as that."

Case 4. "I don't think l've ever got over it. It was dreadful."

Case 5. "It was pretty horrendous, I went through a stage of being really angry but I didn't let it affect my life at all."

Case 6. "I couldn't let my family down. It was a choice of two evils, I did it (late termination at 16 weeks) for my son."

Case 7. Ex-husband reported that this loss had been the most traumatic event that had ever occurred to his ex-wife.

Case 8. "I was devastated. It happened around the time my daughter witnessed me being strangled to the point of unconsciousness by her father."

Case 9. No comments.

Case 10. "It was extremely upsetting, in fact it still is upsetting."

The corresponding comments from the three control mothers included:

Case I."I still get reminders. I had seven weeks off work."

Case 2. (On the first of her three miscarriages.) "I felt quite devastated and inadequate but I was back at work in a fortnight."

Case 3. "It was very upsetting for the first month but I bounced back quite quickly."

\section{Perinatal factors}

Details of birth-related variables for the index subjects and their controls are shown in Table 3. There were no significant differences in either the obstetric management or complication rates. The biggest numerical difference occurred in the frequency of Caesarean sections, with the group with anorexia nervosa having four and the control group having one. Three of these four subjects with anorexia nervosa were cases in which the mothers had experienced stillbirths or early neonatal deaths in their previous pregnancy.

The most notable group difference occurs in the numbers of subjects who were looked after in a SCBU. Again, four out of five of these cases of anorexia nervosa had previously experienced a perinatal death. It seems likely that parental and/or obstetric team anxieties played a part in this.

A larger number of mothers with anorexia nervosa recollected worrying significantly about miscarrying during their pregnancy $(10 / 40 v .2 / 40 ; P=0.028)$. Postnatal maternal depression revealed most index mothers admitting to depressive mood symptoms persisting longer than six months, although this difference did not reach statistical significance. Only one of these index mothers had previously experienced a severe obstetric loss.

\section{Infancy and early childhood}

Fifteen out of $40(37.5 \%)$ index mothers estimated that they provided over $95 \%$ of the baby and infant care, to the almost virtual exclusion of their fathers, compared with only $5 / 40(12.5 \%)$ of the control mothers $(P=0.02)$. Infant sleep pattern difficulties were also significantly overrepresented in the group with anorexia nervosa (19/40 v. 8/40; $P=0.018)$. The index mothers reported experiencing more moderate/severe distress themselves when leaving their daughters in nursery for the first time $(12 / 40 v .4 / 40 ; P=0.048)$. Index mothers reported that their daughters were significantly older when they first spent a weekend in the care of other adults $(P=0.009)$. Highly significant differences were obtained for cut-offs of 11 and 15 years.

Other factors that were enquired about but showed no differences included: the length of time the child was breast-fed; any reported eating difficulties in the first five years; consulting outside professionals in the first two months; the period of time nursing their daughter in the same room as the parents at night; parental perceptions that their daughters needed more care and attention than other children; and the incidence of serious life-threatening illnesses.

\section{Questionnaire results}

A total of $97.5 \%$ (39/40) of the control and $100 \%$ of the index mothers completed the STAI Questionnaire. The mothers scored significantly higher on this measure

Table 3 Birth details of the subjects with anorexia nervosa and control subjects: $\chi^{2}$ group comparisons

\begin{tabular}{|c|c|c|c|}
\hline & Subjects with anorexia nervosa & Control subjects & $P$ \\
\hline \multirow[t]{3}{*}{ Recalled obstetric problems } & $9 / 40$, toxaemia & $10 / 40$, toxaemia & NS \\
\hline & $5 / 40$, antepartum haemorrhage & 2/40 antepartum haemorrhage & NS \\
\hline & $5 / 40$, previous obstetric complications & $\mathrm{I} / 40$, previous obstetric complications & NS \\
\hline \multirow[t]{3}{*}{ Gestation } & $n=36$ full term & $n=37$ full term & NS \\
\hline & $n=336$ weeks & $n=236$ weeks & \\
\hline & $n=132$ weeks & $n=143$ weeks & \\
\hline Forceps deliveries & $9 / 40$ & $\mathrm{II} / 40$ & NS \\
\hline Caesarean sections & $4 / 40$ & $1 / 40$ & NS \\
\hline Mean birth weight & $3.26 \mathrm{~kg}(\mathrm{~s} . \mathrm{d} .=0.46)$ & $3.39 \mathrm{~kg}(\mathrm{~s} . \mathrm{d} .=0.46)$ & NS \\
\hline Apgar Score' at I min of $<7$ & $2 / 40$ & $2 / 40$ & NS \\
\hline Apgar Score' at 5 min of $<7$ & $0 / 40$ & $0 / 40$ & NS \\
\hline \multirow[t]{4}{*}{ Baby looked after in a special care baby unit } & $1 / 40$, part of first day & $2 / 40$, part of first day & NS \\
\hline & $2 / 40$, up to I week & & \\
\hline & I/40, up to 2 weeks & & \\
\hline & I/40, up to 3 weeks & & \\
\hline
\end{tabular}

I. Apgar Score, Apgar (1953). 
(mean $=40.93$ and s.d. $=10.63 v$. mean $=$ 35.26 and s.d. $=7.09 ; P<0.008)$.

\section{Overview of the evidence for high-concern parenting}

In order to determine whether the multiple observed differences occur because a small fraction of the index cases score positively on all high-concern factors, we tabulated the sum total of factors for each subject. The factors included were: severe obstetric losses; fertility worries; significant puerperal worries about losing the pregnancy; near-exclusive maternal child care in the first five years; severe child upset at first regular separation; first weekend away from parents after 11 years of age; and high maternal trait anxiety. Continuously distributed variables were taken to be present if they scored above the control group mean plus one standard deviation. Twenty-three $(57.5 \%)$ of the index cases registered two or more high-concern factors, compared with six $(15 \%)$ of the control cases. The difference in how these factors are distributed between the groups is attested to by the $\chi^{2}$ analysis with the test for trend $(P=0.0002$; see Table 4$)$.

\section{DISCUSSION}

\section{Significant case-control differences in the interview data}

We obtained a wide range of both direct and indirect evidence suggestive of highconcern maternal attitudes and behaviour throughout the early life of the index subjects. Our finding of increased rates of exclusive maternal caring among index mothers in the first five years and the differential rates of spending their first weekend away from parents before the age of $\mathbf{1 1}$ years would seem to fit with a pattern of overprotective parenting classically described by Levy: mothers caring exclusively for a child, excluding external influences, was cited as a central feature of his case series of overprotective mothers (Levy, 1943). This parental behaviour might tend to encourage the development of enmeshed, overly dependent relationships characterised by excessively compliant behaviours in the child, a frequent observation in patients with anorexia nervosa.

Many of the variables that we enquired about relate to dyadic anxiety surrounding separation, suggesting early insecure attachment patterns. The literature suggests that mothers with insecurely attached infants are more likely to have insecure attachment representations themselves (Fonagy et al, 1994) and this attachment classification is known to be linked with higher levels of anxiety disorders in mothers (Manassis et al, 1994).

We found no significant differences in parents' recollections of feeding difficulties in the infants' first five years. This contrasts with Marchi \& Cohen's (1990) findings. Their prospectively obtained evidence for 'picky eating' and 'digestive problems' at their first interview (mean age six years) being associated with anorexic symptoms in adolescence (mean age 16 years) must be interpreted in the light of their random sample of over 800 children actually having no cases of anorexia with amenorrhoea. Their results may not apply to cases of anorexia nervosa as described here.

\section{Maternal trait anxiety}

Our findings of higher index maternal trait anxiety scores are of interest in view of their consistent association with overprotective attitudes (Parker, 1983). Maternal stress or elevated prenatal Spielberger anxiety trait scores have been shown to be associated with increased rates of emergency paediatric consultations in the first years of the infant's life (Goldman \& Owen, 1994). In this prospectively designed study, trait anxiety was the only statistically significant factor to emerge in their regression analyses, with paternal anxiety, obstetric complications, marital satisfaction and life event scores showing no association. Such increased usage of paediatric services would certainly be expected to reflect higher levels of parental concern. In the present study four items targeting evidence of recollected parental concern about their daughters' early physical and developmental health revealed no group differences. Gestational age, birth weight and obstetric complications were not associated with higher trait anxiety scores in this study.

The reported correlation between state and trait anxiety (Spielberger, 1983) invites the interpretation that mothers who have gone through the harrowing experience of their daughter's anorexia may well be made more 'trait' anxious as a result. This might therefore represent the effect of the illness rather than a true premorbid maternal characteristic.

Worrying about the health of the unborn child is likely to be a direct manifestation of parental concern. This experience is likely to relate to both higher constitutional levels of anxiety as well as reflecting a cognitive set influenced by prior aversive experience. Six of the 10 mothers who reported moderate to severe worries about losing their child during pregnancy had STAI scores that were greater than one standard deviation above the group mean. Five had also experienced a severe obstetric loss.

\section{Severe obstetric losses}

In view of these relative methodological merits and deficiencies, the finding of a high frequency of previous severe obstetric losses experienced by the index parents prior to the birth of their daughters, although just below the threshold for significance $(P=0.066)$, is of interest. The significance of this difference is increased by the observation that nine of the 10 daughters in this subgroup were the next-born child after their parent's tragic loss.

There is evidence from the paediatric literature that the child born immediately after a tragic loss may become the recipient

I. Cell totals were aggregated to ensure that cell values exceeded 5 .

2. $\chi^{2}=\mid 7.21$; d.f. $=2$. 
of parental overprotective care (Levy, 1943). This phenomenon has been referred to as 'the vulnerable child syndrome' (Green \& Solnit, 1964) or 'the replacement child syndrome' (Powell, 1995). One descriptive report has linked a subgroup of young children with anorexia nervosa specifically with the vulnerable child syndrome (Atkins \& Silber, 1993). Early pregnancy following a stillbirth has been shown to be associated with increased rates of maternal depression and anxiety (Hughes et al, 1999).

It is notable that in Rastäm \& Gillberg's (1992) population-based study of anorexia nervosa they report a higher than expected prevalence of deceased first-degree relatives in their sample of 52 community-detected cases. They found four cases of siblings who had died before the onset of anorexia nervosa, but no sibling deaths in their control group. They do not report any data regarding their subjects' mothers' previous obstetric histories. Both clinically and in the research literature (Kirkley-Best \& Kellner, 1982), it is noted how perinatal losses and miscarriages are frequently under-reported, hence this figure may be an underestimate.

\section{Differences in obstetric complications}

In respect of our second hypothesis we found no evidence for an excess of obstetric complications, prematurity, neonatal distress or smaller birth weights in the births of our subjects with anorexia nervosa. This accords with one population-based study (Rastäm, 1992) but contrasts with one uncontrolled (Morgan \& Russell, 1975) and one controlled study (Kay et al, 1967). Our finding of a slight excess of cases looked after in SCBUs (five anorectic cases compared to two controls) may be a reflection of the maternal (and probably medical) anxiety relating to the earlier loss of a viable child in four of these five anorectic cases.

\section{Methodological merits of data sources}

The medical case note data are likely to be the most robust data because they are not susceptible to any form of systematic parental recall bias. The semi-structured interview data are likely to be of intermediate reliability and validity because they concentrate on the mother's recollections of her pregnancy and the child's early infancy

\section{CLINICAL IMPLICATIONS}

- Parental high concern in anorexia nervosa does appear to antedate the onset of the illness.

Sympathetic enquiries about parental experiences that might lead to early heightened concern and expectation should be included in the routine clinical assessment of these cases.

Explicitly acknowledging the early effects of these factors may help families move away from these dynamics.

\section{LIMITATIONS}

Maternal interviews were not conducted blind to group membership.

- The only externally corroborated data source was that obtained from obstetric and maternity notes.

- The study's methodology does not eliminate the possible effect of recall bias for some of the high-concern factors analysed.

PHILIP J. SHOEBRIDGE, MRCPsych, North Bristol NHS Trust, Southmead Hospital, Bristol; SIMON G. GOWERS, FRCPsych, University of Liverpool, Liverpool

Correspondence: Dr P. Shoebridge, Consultant Child, Adolescent and Family Psychiatrist, Southmead Hospital,Westbury-on-Trym, Bristol BSI0 5NB

(First received 17 May 1999, final revision 9 September 1999, accepted 10 September 1999)

during a period clearly distinct from any prodromal or active anorexic illness behaviour. Nevertheless, reattributions and reinterpretations of these aspects of the premorbid family environment may be made in an 'effort after meaning' in the light of the profound upset caused by their daughter's illness.

\section{Methodological strengths and weaknesses}

One of the methodological deficiencies of our study is that the maternal interviews were not conducted blind to group membership. The method of administering the interview, with its structured format and standardised manner of asking questions and recording answers, should limit tendencies for bias by the interviewer, although this possibility cannot be excluded. The procedure of anonymising the subject interviews prior to a research colleague quantifying the qualitative aspects of the interviewees' responses did permit single- blind ratings of many of the variables from this data source.

A further limitation in common with much previous research in this area is the modest size of the sample, increasing the likelihood of Type I error. In addition, despite opting for more conservative statistical tests (using group rather than pairwise matching), we have not employed Bonferroni's correction in view of the large number of comparisons made. While making these points, it is worth drawing attention to the fact that the sample sizes in this study are relatively substantial when viewed in the context of previous similar research. Being a rare disorder, accumulating large, diagnostically pure samples of subjects with anorexia nervosa requires multicentre collaboration or substantial recruitment periods (Shaw \& Garfinkel, 1990). Indeed, the implication of this study finding statistically significant differences in such relatively small sample sizes, taken together with the multiple trends that occurred in the anticipated direction, suggests that parental high concern may have a substantial 
effect size as a factor contributing to the aetiology of anorexia nervosa. It is also relevant that we found such a variety of significant group differences, despite the close matching of the study and control groups, because overmatching can reduce the variability between the samples of the factor(s) of primary interest (Schlesselman, 1982).

Among the strengths of the study are the low refusal rates, a consecutive sample of cases of anorexia nervosa, the wellmatched samples, the high percentage of case notes that were traced and the combination of convergent strategies, all of which separately give evidence supporting the central hypotheses. The inclusion of some qualitative data about the parents' recollections of their losses may be seen as enriching the data.

This sample of girls with anorexia nervosa was recruited from a regional referral centre, which might attract a relatively severely affected clinical group. Hence, conclusions derived from these findings may not be safely generalised to adolescents presenting with less serious disorders.

\section{REFERENCES}

American Psychiatric Association (1987) Diagnostic and Statistical Manual of Mental Disorders (3rd edn, revised) (DSM-III-R). Washington, DC: American Psychiatric Association.
Apgar, V. (1953) A proposal for a new method of evaluation of the newborn infant. Current Researches in Anesthesia and Analgesia, 32, 260-267.

Atkins, D. M. \& Silber, T. J. (1993) Clinical spectrum of anorexia nervosa in children. Journal of Developmental and Behavioural Pediatrics, 14, 211-216.

Fonagy, P., Steele, M., Steele, H., et al (1994) The theory and practice of resilience. Journal of Child Psychology and Psychiatry, 35, 231-257.

Forfar, J. O. (1984) Demography, vital statistics and the patterns of disease in childhood. In Textbook of Paediatrics (eds J. O. Farfar \& G. C. R. O'Neil). Edinburgh: Churchill Livingstone.

Garner, D. M., Olmstead, M. P., Bohr, Y., et al (1982)

The Eating Attitudes Test: psychometric features and clinical correlates. Psychological Medicine, 12, 87I-878.

Goldman, S. L. \& Owen, M. T. (1994) The impact of parental trait anxiety on the utilisation of health care services in infancy: a prospective study. Journal of Paediatric Psychology, 19, 369-381.

Green, M. \& Solnit, A. J. (1964) Reactions to the threatened loss of a child: a vulnerable child syndrome. Pediatrics, 34, 58-66.

Hughes, P. M., Turton, P. \& Evans, C. D. H. (1999) Stillbirth as a risk factor for depression and anxiety in the next pregnancy: cohort study. British Medical Journal, 318, 1721-1724.

Kay, D.W. K., Schapira, K. \& Brandon, S. (1967) Early factors in anorexia nervosa compared to non-anorexia groups. Journal of Psychosomatic Research, II, I33-139.

Kirkley-Best, E. \& Kellner, K. (1982) The forgotten grief: a review of the psychology of still birth. American journal of Orthopsychiatry, 52, 420-429.

Levy, D. M. (1943) Maternal Overprotection. Columbia: Columbia University Press.

Manassis, K., Bradley, S., Goldberg, S., et al (1994) Attachment in mothers with anxiety disorders and their children. Journal of the American Academy of Child and Adolescent Psychiatry, 33, II06-1II3.
Marchi, M. \& Cohen, P. (1990) Early childhood eating behaviours and adolescent eating disorders. Journal of the American Academy of Child and Adolescent Psychiatry, 29 II2-117.

Market Research Society (199I) Occupational Groups: a Job Dictionary (3rd edn). London: Market Research Society.

Morgan, H. G. \& Russell, G. F. M. (1975) Value of family background and clinical features as predictors of long-term outcome in anorexia nervosa: four-year follow-up study of $4 \mathrm{I}$ patients. Psychological Medicine, 5 355-37।.

Parker, G. (1983) Parental Overprotection. A Risk factor in Psychosocial Development. Sydney: Grune \& Stratton.

Powell, M. (1995) Sudden infant death syndrome: the subsequent child. British Journal of Social Work, 25, 227-240.

Rastäm, M. (1992) Anorexia nervosa in 51 Swedish adolescents: premorbid problems and comorbidity Journal of the American Academy of Child and Adolescent Psychiatry, 31, 819-829.

— \& Gillberg, C. (1992) Background factors in anorexia nervosa: a controlled study of $5 \mathrm{I}$ teenage cases including a population sample. Journal of the American Academy of Child and Adolescent Psychiatry, 3I, 819-829.

Schlesselman, J. J. (1982) Case-Control Studies. Design Conduct, Analysis. Oxford: Oxford University Press.

Shaw, B. \& Garfinkel, P. (1990) Research problems in the eating disorders. International Journal of Eating Disorders, 9, 545-555.

Spielberger, C. D. (1983) Manual for the State-Trait Anxiety Inventory. Palo Alto, CA: Consulting Psychologists Press.

Wood, A., Waller, G., Miller, J., et al (1992) The development of Eating Attitude Test scores in adolescence. International Journal of Eating Disorders, II, 279-282. 